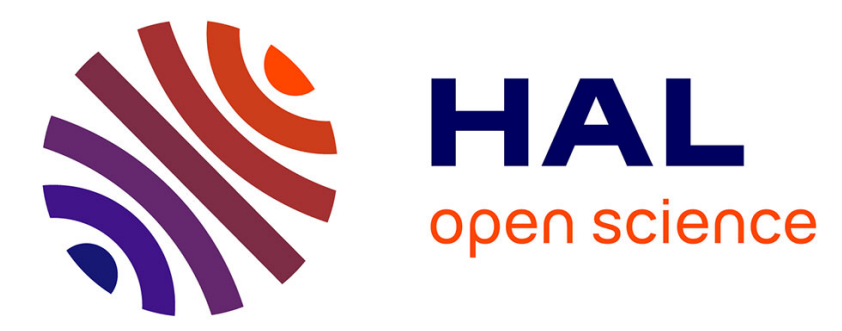

\title{
Estimation of adsorption-induced pore pressure and confinement in a nanoscopic slit pore by a density functional theory
}

David Grégoire, Carine Malheiro, Christelle Miqueu

\section{- To cite this version:}

David Grégoire, Carine Malheiro, Christelle Miqueu. Estimation of adsorption-induced pore pressure and confinement in a nanoscopic slit pore by a density functional theory. Continuum Mechanics and Thermodynamics, 2018, 30 (2), pp.347-363. 10.1007/s00161-017-0602-x . hal-01637875

\section{HAL Id: hal-01637875 \\ https://hal.science/hal-01637875}

Submitted on 18 Nov 2017

HAL is a multi-disciplinary open access archive for the deposit and dissemination of scientific research documents, whether they are published or not. The documents may come from teaching and research institutions in France or abroad, or from public or private research centers.
L'archive ouverte pluridisciplinaire HAL, est destinée au dépôt et à la diffusion de documents scientifiques de niveau recherche, publiés ou non, émanant des établissements d'enseignement et de recherche français ou étrangers, des laboratoires publics ou privés. 


\title{
Estimation of adsorption-induced pore pressure and confinement in a nanoscopic slit pore by a density functional theory
}

\author{
David Grégoire • Carine Malheiro • \\ Christelle Miqueu
}

Received: date / Accepted: date

\begin{abstract}
This study aims at characterising the adsorption-induced pore pressure and confinement in nanoscopic pores by molecular non-local density functional theory (DFT). Considering its important potential industrial applications, the adsorption of methane in graphitic slit pores has been selected as the test case. While retaining the accuracy of molecular simulations at pore scale, DFT has a very low computational cost that allows obtaining highly resolved pore pressure maps as a function of both pore width and thermodynamic conditions. The dependency of pore pressure on these parameters (pore width, pressure and temperature) is carefully analysed in order to highlight the effect of each parameter on the confined fluid properties that impact the solid matrix.
\end{abstract}

Keywords pore pressure $\cdot$ adsorption $\cdot$ microporous $\cdot$ density functional theory methane $\cdot$ carbon

\section{Introduction}

Following the IUPAC recommendation [1,2, the pore space in porous materials is divided into three groups according to the pore size diameters: macropores of widths greater than $50 \mathrm{~nm}$, mesopores of widths between 2 and $50 \mathrm{~nm}$ and micropores ${ }^{1}$ of widths less than $2 \mathrm{~nm}$. Zeolites, activated carbons, tight rocks, coal

C. Miqueu and D. Grégoire conceived the research goals, hypothesis and methodology and contributed equally.

University Pau \& Pays Adour,

Laboratoire des Fluides Complexes et leurs Réservoirs, LFCR-IPRA, UMR5150,

Campus Montaury, F-64600 Anglet, France

Tel.: +33-5-5957-4479

Fax: +33-5-5957-4439

Corresponding authors: C. Miqueu \& D. Grégoire

E-mail: christelle.miqueu@univ-pau.fr \& david.gregoire@univ-pau.fr

D. Grégoire is fellow of the Institut Universitaire de France.

1 The term nanopores is sometimes preferred in the Mechanics community to label pores of width less than $2 \mathrm{~nm}$. However, the 2015 IUPAC report [2] recommends that this term embraces the three categories of pores, but with an upper limit $\approx 100 \mathrm{~nm}$. 
rocks, source rocks, cement paste or construction materials are among microporous materials. In recent years, a major attention has been paid on these microporous materials because the surface-to-volume ratio (i.e., the specific pore surface) increases with decreasing characteristic pore size. Consequently, these materials can trap an important quantity of fluid molecules as an adsorbed phase. This is important for applications in petroleum and oil recovery, gas storage, separation, catalysis or drug delivery.

In very small pores, the molecules of fluid are confined (molecular packing). This effect induces that fluid-fluid and fluid-solid interactions sum at the pore scale and has significant consequences at the macroscale, such as instantaneous swelling or shrinkage deformation. In different contexts, these deformations may be critical. A lot of natural and synthesised porous media are composed of a double porosity: the microporosity where the fluid is trapped as an adsorbed phase and a meso or a macro porosity required to ensure the transport of fluids to and from the smaller pores. If adsorption in nanopores induces instantaneous deformations at a higher scale, the matrix swelling may close the transport porosity, reducing the global permeability of the porous system or annihilating the functionality of synthesised materials. Particularly, in situ adsorption-induced coal swelling has been identified [3, 4] as the principal factor leading to a rapid decrease in $\mathrm{CO}_{2}$ injectivity during coal bed methane production enhanced by $\mathrm{CO}_{2}$ injection. Conversely, gas desorption can lead to matrix shrinkage and microcracking, which may help oil and gas recovery in the context of unconventional petroleum engineering 5 . The effects of adsorbent deformation on physical adsorption has also been identified [6] as the major challenge concerning gas porosimetry in nano-porous non-rigid materials (e.g. metal organic frameworks). In conclusion, there is now a consensus in the community that major attention has to be focused on the coupled effects appearing at the nanoscale within microporous media because they may have significant consequences at the macroscale [7].

Experimentally, different authors tried to combine gas adsorption results and volumetric swelling data (see e.g. [7] for a review). Pioneer works of Meehan [8] showed the effect of carbon dioxide sorption on the expansion of charcoal but only mechanical deformations were reported and no adsorption quantities were measured. Later on, different authors [9,5, 10, 11, 12, 13, 14] highlighted the phenomenon on bituminous coals, because it is of utmost importance in the context of $\mathrm{CO}_{2}$ geological sequestration and coal bed reservoirs exploitation. Recently, Perrier et al. [15, 16] performed simultaneous measurements of adsorbed quantities and induced strain on an activated carbon and showed that the volumetric swelling is directly linked to the excess adsorbed quantity.

As far as modeling is concerned, standard poromechanics [17,18 links the macroscopic strain to the total stress and the pore fluid pressure (i.e. the bulk pressure in that case) and has led to relevant descriptions of various multiphysics phenomena in macroporous materials. However, this classical approach fails in describing adsorption-induced strain and predicts volumetric shrinkage instead of volumetric swelling for these microporous materials [19. Such classical framework needs to be extended to take into account the fluid confinement effects present within the nanopores. Especially, molecular packing induces a deviation of the pore fluid pressure from its bulk value, the difference being most often denoted as solvation force or pressure [20. The effect of this modified pore fluid pressure on the pore walls has to be characterized at the pore scale and upscaled to rep- 
resent macroscopic swelling strain. Molecular simulations are the classical tools to represent the distribution of molecules due to fluid-fluid and fluid-solid interactions at the nanoscale. Different authors used Monte Carlo simulation tools to study the pore fluid pressure profiles within nanopores. Do and coworkers performed Grand Canonical Monte Carlo simulations for argon 21,22, methane and methanol 23] in slit-shaped micropores with movable solid layers to compute the pore pressures and corresponding deformations. Kowalczyk and coworkers studied adsorption-induced deformation of microporous carbons filled with carbon dioxide [24,25] and near-critical argon [26. These investigations showed that pressures applied on the pore surfaces may be very high (few hundred of MPa), depending on the thermodynamic conditions and on the pore sizes. However, even if macroscopic adsorption isotherms may be reconstructed in a consistent way from molecular simulations through the material pore size distribution [27, molecular simulations are not a tractable nor an efficient tool to predict resulting deformations at a macroscale in a complex material. Hence, enhanced poromechanics framework have been developed [19,28, 16,29] to obtain semi-analytical efficient and tractable tools capable to predict macroscopic strain induced by adsorption in homogeneous microporous materials. In these formulations, the fluid confinement is macroscopically deduced from experimental measurements of adsorbed quantities and not from the thermodynamic state of the fluid at the nanoscale. For micro-to-macro heterogeneous porous media, the fluid confinement cannot be deduced from experimental measurement and new strategies have to be proposed.

If molecular simulations are too time consuming to be coupled with poromechanical models or macroscopic numerical simulations, an elegant and relevant alternative approach consists in estimating the fluid confinement with a molecular density functional theory $(\mathrm{DFT})^{2}$. It has been already shown 32,33 that nonlocal DFT is as accurate as molecular simulations to estimate density profiles of real fluids in nanopores but with a very lower computational cost. Another key feature is that DFT is written in a continuum framework and then may be coupled more easily with continuum poromechanics theory than discrete simulations results. Balbuena et al. 34 proposed a theoretical study based on DFT to estimate the solvation pressure of Lennard-Jones fluids in slit micropores. At low pressure and low temperature conditions, Neimark and co-workers [35, 36, 37. or Ustinov and Do 38 used different DFT variations to estimate the solvation pressure (e.g. for porous media characterisation purposes). To our knowledge, only one article in the literature proposes to apply this strategy on a large range of temperature and pressure 39 , by studying the adsorption of methane on natural coals at geological conditions.

This paper aims at using a non-local density functional theory combined with a powerful equation-of-state in order to obtain highly-resolved pore pressure maps as a function of bulk pressure and pore width at different temperatures. The proposed method allows to better characterise the complex pore pressure behaviour for large thermodynamic conditions on the whole range of micropore widths even for complex fluids. This is the first step to provide a unified theory from nano to macro scales that links adsorption of the fluid in the nanopores to the macroscopic deformation of the material.

${ }^{2}$ A comprehensive description of DFT and its applications can be found in the review of Evans [30] or the monograph of Davis 31. 


\section{Theory}

\subsection{Brief overview of NLDFT}

The version of the Non-Local Density Functional Theory (NLDFT) used in this work has been fully described in Malheiro et al. 32. Therefore, the discussion on this theory will be limited to its most significant features here.

Let us consider a fluid of chemical potential $\mu$ at temperature $T$ in a volume $V$. In the density functional theory (DFT) framework [30, the grand thermodynamic potential $\Omega$ of an inhomogeneous fluid in an external field in the grand canonical ensemble $(\mu, V, T)$ is given by:

$$
\Omega[\underline{\rho}]=A[\underline{\rho}]-\sum_{i=1}^{N} \int_{V} \rho_{i}(\underline{x})\left[\mu_{b i}-V_{i}^{e x t}(\underline{x})\right] d x .
$$

In Eq. (1), $\Omega$ is the grand thermodynamic potential, $A$ is the Helmholtz free energy, $\underline{\rho}=\left(\rho_{1}, \ldots, \rho_{N}\right)$ is the vector of the different molecular densities of the $N$ species of the inhomogeneous fluid, which depend on the position vector $\underline{x}, V_{i}^{\text {ext }}$ is an external field (e.g. a fluid/solid interaction potential), $\mu_{b i}$ is the bulk chemical potential of the specie $i$.

The minimization of this grand potential leads to the Euler-Lagrange equations that allow computing the density distributions of the fluid at equilibrium:

$$
\frac{\delta \bar{\Omega}[\underline{\rho}]}{\delta \rho_{i}}=\frac{\delta \bar{A}[\underline{\rho}]}{\delta \rho_{i}}-\mu_{b i}+V_{i}^{e x t}=0 \quad \forall i \in[1, N] .
$$

In Eq. $(2),(\bar{\Omega}, \bar{A}$ are the volumetric grand thermodynamic potential and the volumetric Helmholtz free energy respectively.

In this work, the SAFT-VR equation of state [40] has been used as the thermodynamic model to compute both the properties of the bulk fluid phase and the chemical potential at equilibrium and as the bulk limit to build the inhomogeneous Helmholtz free energy. In this work, we deal with methane, a fluid of non-associating spherical monomers of diameter $\sigma$, with a mean-field (MF) approximation, i.e. the correlations due to the long-range attractive interactions are neglected. In that case, the Helmholtz free energy functional is given by:

$$
A[\underline{\rho}]=\int_{V} \bar{A}[\underline{\rho}(\underline{x})] d x=\underbrace{A^{\text {ideal }}[\rho]}_{\begin{array}{c}
\text { ideal } \\
\text { part }
\end{array}}]+\underbrace{A^{\mathrm{HS}}[\underline{\rho}]}_{\begin{array}{c}
\text { hard sphere } \\
\text { contribution }
\end{array}}+\underbrace{A_{1}^{\mathrm{lr}}[\underline{\rho}]+A_{1}^{\mathrm{sr}}[\underline{\rho}]+A_{2}[\underline{\rho}]}_{\text {dispersive part }} .
$$

In Eq. (3):

$-A^{\text {ideal }}$ is the ideal part and is exactly known as:

$$
A^{\text {ideal }}[\underline{\rho}]=k_{B} T \sum_{i=1}^{N} \int_{V} \rho_{i}(\underline{x})\left(\ln \left[\rho_{i}(\underline{x}) \Lambda_{i}^{3}\right]-1\right) d x .
$$

In Eq. (4), $k_{B}$ is the Boltzmann constant and $\Lambda_{i}$ is the thermal de Broglie wavelength. 
- The hard sphere contribution $A^{\mathrm{HS}}$ is computed non locally with the modified fundamental measure theory (MFMT) [4, 42, taking into account scalar and vectorial contributions:

$$
A^{\mathrm{HS}}[\underline{\rho}]=k_{B} T \int_{V}\left(\Phi^{\mathrm{HS}, \mathrm{s}}[\underline{\rho}(\underline{x})]+\Phi^{\mathrm{HS}, \mathrm{v}}[\underline{\rho}(\underline{x})]\right) d x
$$

with

$$
\left\{\begin{array}{l}
\Phi^{\mathrm{HS}, \mathrm{s}}=-n_{0} \ln \left(1-n_{3}\right)+\frac{n_{1} n_{2}}{1-n_{3}}+\frac{n_{2}^{3} \ln \left(1-n_{3}\right)}{36 \pi n_{3}^{2}} \\
\Phi^{\mathrm{HS}, \mathrm{v}}=\frac{\underline{n}_{4} \underline{n}_{5}}{1-n_{3}}-\frac{n_{2} \underline{n}_{5} \underline{n}_{5} \ln \left(1-n_{3}\right)}{12 \pi n_{3}^{2}}-\frac{n_{2} \underline{n}_{5} \underline{n}_{5}}{12 \pi n_{3} \ln \left(1-n_{3}\right)^{2}}
\end{array}\right.
$$

and

$$
\begin{cases}\forall \alpha \in[0,3], & n_{\alpha}[\underline{\rho}(\underline{x})]=\sum_{i=1}^{N} \int_{V} \rho_{i}(\underline{\xi}) w_{\alpha}(\|\underline{x}-\underline{\xi}\|) d \xi \\ \forall \beta \in[4,5], & \underline{n}_{\beta}[\underline{\rho}(\underline{x})]=\sum_{i=1}^{N} \int_{V} \rho_{i}(\underline{\xi}) w_{\beta}(\underline{x}-\underline{\xi}) d \xi\end{cases}
$$

where

$$
\left\{\begin{array}{l}
w_{0}(r)=\frac{1}{\pi \sigma^{2}} \delta\left(\frac{\sigma}{2}-r\right), w_{1}(r)=\frac{1}{2 \pi \sigma} \delta\left(\frac{\sigma}{2}-r\right), w_{2}(r)=\delta\left(\frac{\sigma}{2}-r\right), \\
w_{3}(r)=\mathcal{H}\left(\frac{\sigma}{2}-r\right), \quad w_{4}(\underline{r})=\frac{\underline{r}}{2 \pi \sigma r} \delta\left(\frac{\sigma}{2}-r\right), w_{5}(\underline{r})=\frac{r}{r} \delta\left(\frac{\sigma}{2}-r\right) .
\end{array}\right.
$$

In Eq. (8), $\delta$ and $\mathcal{H}$ are respectively the Dirac and the Heaviside functions.

- The dispersive part encompasses a first-order and a second-order monomer perturbation. The first-order monomer perturbation has been partitioned to get on the one hand the "short-range" contribution $A_{1}^{\mathrm{sr}}$ and on the other hand the "long-range" contribution $A_{1}^{\mathrm{lr}}$ that becomes the classical attractive term of the DFT framework in the mean-field approximation [43]. We get

$$
A_{1}^{\mathrm{lr}}[\underline{\rho}]=\frac{1}{2} \sum_{i=1}^{N} \sum_{j=1}^{N} \int_{V} \rho(\underline{x})\left(\int_{V} \rho(\underline{\xi}) \Phi_{i j}^{\mathrm{att}}(\|\underline{x}-\underline{\xi}\|) d \xi\right) d x .
$$

In Eq. $(9), \Phi_{i j}^{\text {att }}$ is the attractive part of the square-well potential. The "shortrange" contribution $A_{1}^{\mathrm{sr}}$ and the second-order monomer perturbation $A_{2}$ are estimated through a coarse-grained approach (see 32 for full details) from a weighted density:

$$
\tilde{\rho}(\underline{x})=\frac{3}{4 \pi \sigma^{3}} \int_{V} \rho(\underline{\xi}) \mathcal{H}(\sigma-\|\underline{x}-\underline{\xi}\|) d \xi .
$$

In the following, we are considering a slit pore of width $L$ made of two solid parallel graphitic surfaces and filled with methane (see Fig. 1). The external potential $V^{e x t}$ applied on the fluid is the sum of the Steele's interaction potentials [44]:

$$
\left\{\begin{aligned}
V^{\text {ext }}(z) & =V^{\text {steele }}(z)+V^{\text {steele }}(L-z) \\
V^{\text {steele }}(z) & =2 \pi \varepsilon_{s f} \rho_{s} \sigma_{s f}^{2} \Delta\left[0.4\left(\frac{\sigma_{s f}}{z}\right)^{10}-\left(\frac{\sigma_{s f}}{z}\right)^{4}-\left(\frac{\sigma_{s f}^{4}}{3 \Delta(z+0.61 \Delta)^{3}}\right)\right]
\end{aligned}\right.
$$




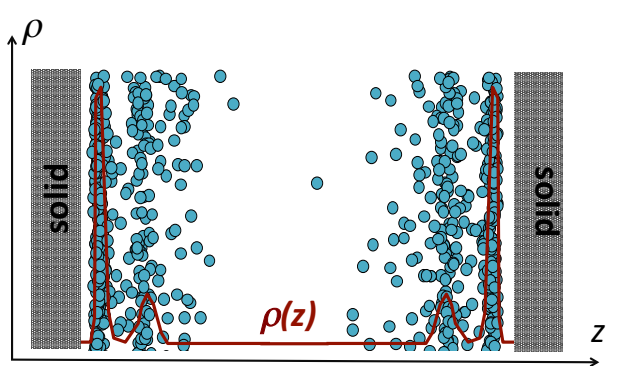

Fig. 1 Schematic view of a slit pore filled with methane.
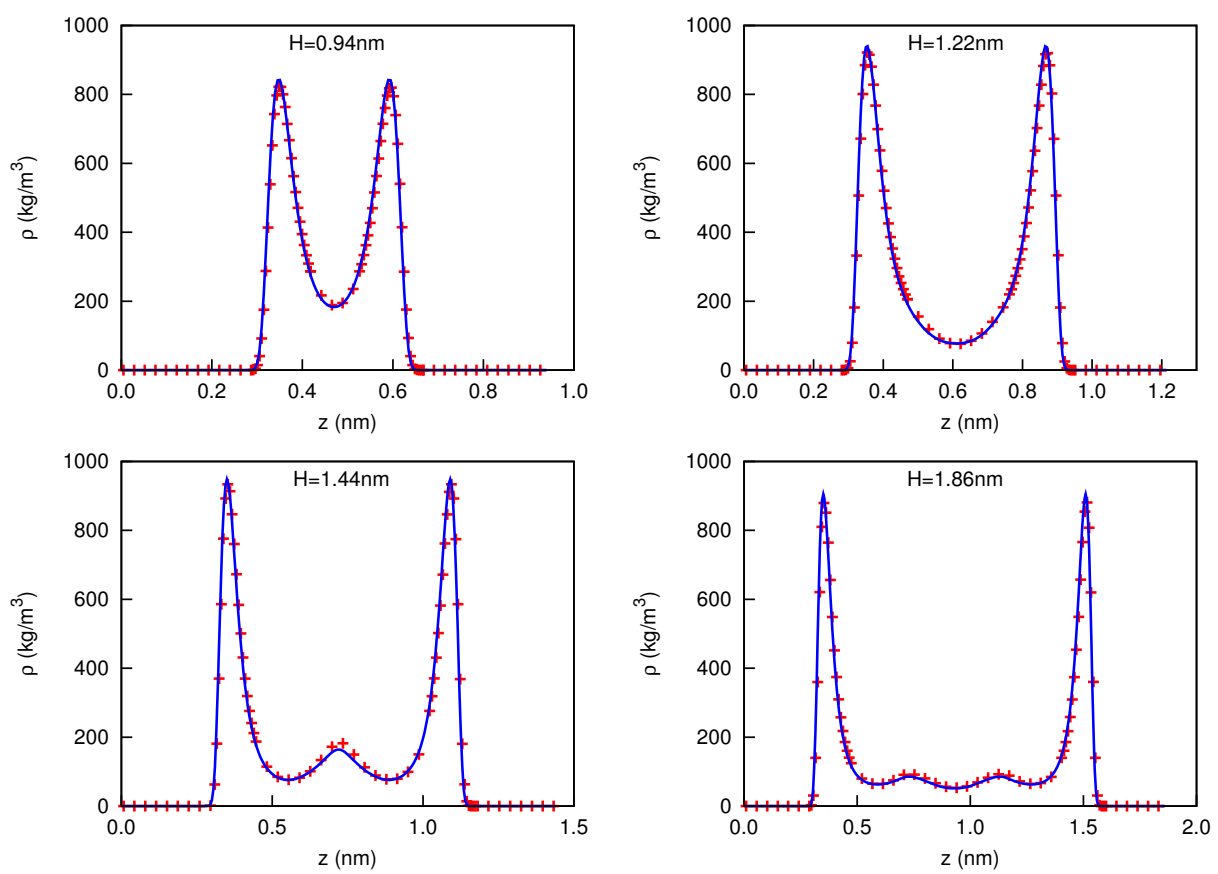

Fig. 2 Density profiles of methane in carbon slit pores at $T=353 \mathrm{~K}$ and $P=6 \mathrm{MPa}$ with different pore sizes. (lines): NLDFT modeling; (symbols) : GCMC molecular simulations (Adapted from 32]).

In Eq. 11), $\rho_{s}=114$ molecules $/ \mathrm{m}^{3}$ is the density of graphite, $\Delta=0.335 \mathrm{~nm}$ is the space between two layers of graphite constituting the wall [45. $(\sigma, \varepsilon)$ are respectively the collision diameter and the depth of the well potential. $\sigma_{s f}$ and $\varepsilon_{s f}$ are the solid-fluid interaction parameters determined by the Lorentz-Berthelot rules $\sigma_{s f}=\frac{\sigma_{s s}+\sigma_{f f}}{2}$ and $\varepsilon_{s f}=\sqrt{\varepsilon_{s s} \varepsilon_{f f}} \cdot\left(\sigma_{f f}, \varepsilon_{f f}\right)$ are the fluid monomer parameters in the SAFT-VR theory and $\left(\sigma_{s s}, \varepsilon_{s s}\right)$ are the solid parameters.

As shown in Malheiro et al. [32, and reprinted in Fig. 2, molecular simulations have been used to validate the methane's density profiles obtained with the NLDFT framework presented above in the full micropores range. 


\subsection{Pore pressure estimation}

The grand thermodynamic potential of a fluid confined in a slit-like pore of width $L$ between two parallel plates of surface $A_{0}$ being [46]:

$$
d \Omega=-S d T-\sum_{i} N_{i} d \mu_{i}+2 \gamma d A_{0}-\Pi_{f} A_{0} d L
$$

the pressure $\Pi_{f}$ is:

$$
\Pi_{f}=-\frac{1}{A_{0}}\left(\frac{\partial \Omega}{\partial L}\right)_{T, A_{0}, \mu_{i}} .
$$

Thus defined, $\Pi_{f}$ becomes equal to the bulk pressure $P_{b}$ when $L \rightarrow \infty$. It is the sum of two contributions $\Pi_{f}=P_{b}+f_{s}, f_{s}$ being the solvation pressure as introduced by Hansen and McDonald 46] that vanishes when $L \rightarrow \infty$. One can demonstrate (see Appendix A for a complete development) from Eqs. (1) and (13) that:

$$
\Pi_{f}=-\int_{0}^{L} \rho(z) \frac{\partial V^{e x t}(z, L)}{\partial L} d z .
$$

In case of a Steele-type external potential (see Eq. [11), we get also:

$$
\Pi_{f}=-\int_{0}^{L} \rho(z) \frac{\partial V^{\text {steele }}}{\partial z}(z) d z
$$

Eq. (15) is in agreement with Eq. (6.5.5) of Hansen and McDonald's book 46] or 34. Thus, the pressure inside a slit-like pore can be computed with Eq. 15 as soon as the density profiles have been computed with the NLDFT.

\section{Results and discussion}

The pore pressure $\Pi_{f}$ has been computed in the whole range of micropores ( $L<2 \mathrm{~nm}$ ), for pressures up to $20 \mathrm{MPa}$ at three temperatures : 303,373 and $443 \mathrm{~K}$. As an example, the $3 \mathrm{D}$ map of $\Pi_{f}=f\left(L, P_{b}\right)$ at $373 \mathrm{~K}$ is plotted in Fig. 3 whereas the $3 \mathrm{D}$ maps corresponding to the other temperatures are plotted in appendix B (Fig. 10). Animated versions of these 3D maps are given as supplementary materials.

These maps $\Pi_{f}=f\left(L, P_{b}\right)$ clearly highlight that the pore pressure is a complex function of both pore size and thermodynamic conditions and that its variation is far from being obvious. Indeed, it depends on an intricate interplay between fluid-solid and fluid-fluid interactions; the first ones being highly dependent on the distance between pore walls (given an adsorbent-adsorbate pair) and the latest ones on the thermodynamic conditions (pressure and temperature). As already observed in some molecular simulations [21,23] and DFT [35,39] calculations in idealised slit or cylindrical pores, the pore pressure oscillates as a function of pore width as a result of commensurate or incommensurate packing. As mentioned in the Introduction, this behaviour has already been presented in the literature with molecular simulations. However, the calculation speed of thermodynamic theories 


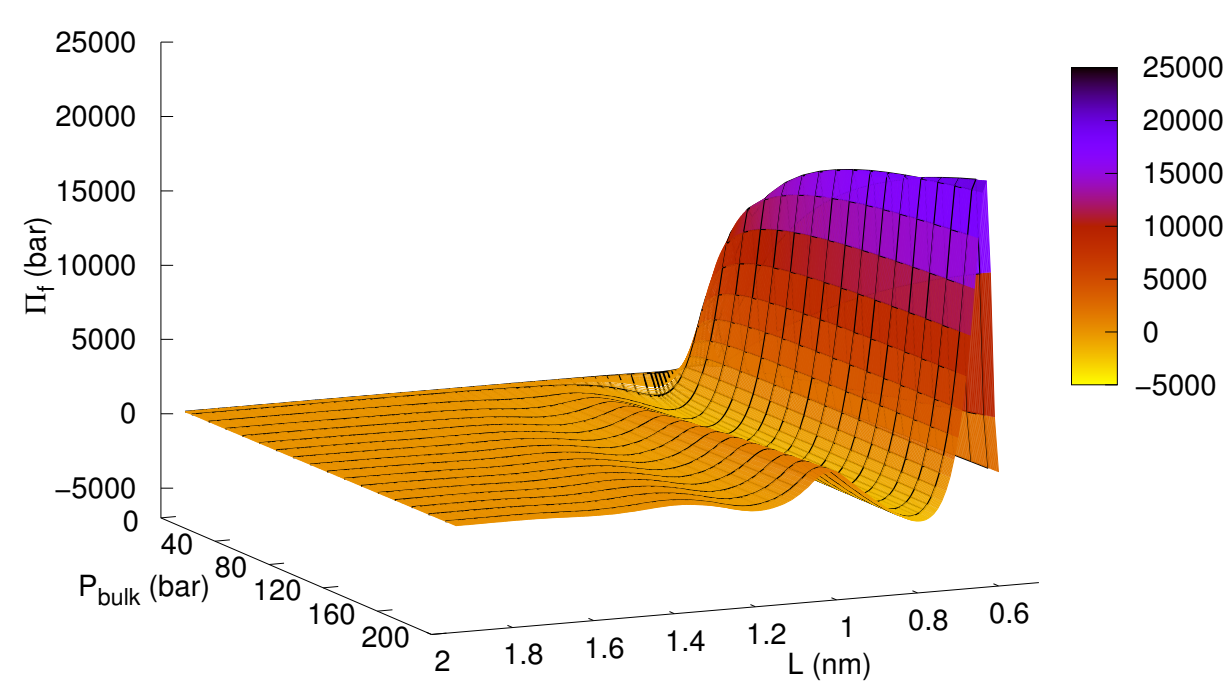

Fig. 3 Pore pressure as a function of bulk pressure and pore size at $373 \mathrm{~K}$.

such as NLDFT compared to molecular simulations allows drawing complete maps of pore pressure for wide thermodynamic conditions and pore ranges. Pore pressures can reach extremely high values in the ultramicropores range. For the largest micropores, the difference between the pore and bulk pressures becomes negligible, the pores being filled with methane at a nearly bulk state, except for the layers on contact with the solid walls. Moreover, the pore pressure successively increases or decreases with bulk pressure depending on the considered pore width range, as better illustrated in the 2D plots of Fig. 4 . As shown in Fig. 4 for $L<0.72 \mathrm{~nm}$, the pores exhibit only positive pore pressure, and so positive adsorption stress causing expansion. This range of widths corresponds to pores able to accommodate only one fluid layer. On the contrary, for $L>0.72$, i.e. for pores accommodating at least two overlapping layers, one can observe a transition from initial negative pore pressure to positive ones with increasing bulk pressures. This behaviour reflects the competition between fluid-solid interactions predominant at low pressures and fluid-fluid interactions that dominate at the highest pressures. Finally, one can see in Fig. 4 that, at a given bulk pressure, $\Pi_{f}$ successively oscillates as a function of pore width, thus reflecting packing effects for such parallel ideal solid surfaces. This behaviour is better illustrated in Fig. 5 where $\Pi_{f}$ is plotted as a function of pore width at different bulk pressures at $T=373 \mathrm{~K}$.

As can be seen in Fig. 5 , when $L$ increases, the pore pressure exhibits an oscillatory decay similar to the one of the pair correlation function, giving successively repulsive or attractive force on the surface of the solid walls. Even if the oscillations of $\Pi_{f}$ reflect the layers formation in the case of infinite parallel smooth walls, the same behavior has been observed in slit micropores of finite length 47] or of heterogeneous surface [39]. For the largest micropores $(L>1.5 \mathrm{~nm})$, the packing effects become negligible and there is no more additional stress applied on the surfaces. A quantitative comparison cannot be done with the previous results of Kopanichuk et al. 47] and Yang et al. 39, due to the differences between both the fluid and the solid representations. However, the solvation pressure evolutions pre- 

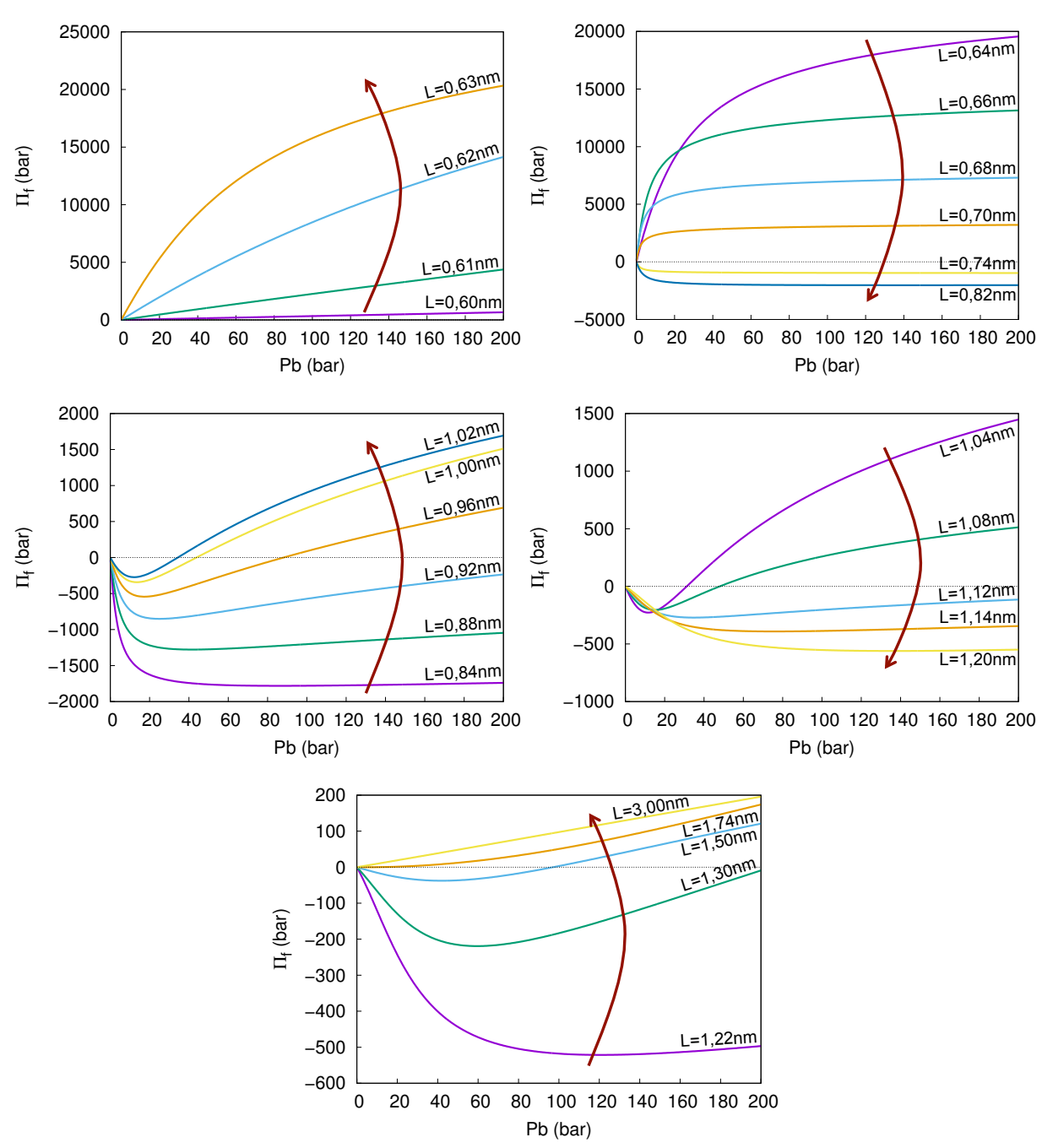

Fig. 4 Pore pressure computed with Eq. 15 as a function of bulk pressure at $373 \mathrm{~K}$ depicted for various pore ranges.

sented here have features in perfect qualitative agreement with the works of these authors. Indeed, on the one hand, Kopanichuk et al. performed GCMC simulations in finite length graphitic slit pores. They obtained the same oscillatory but less pronounced behavior of the solvation pressure because, as they mentioned, fluid adsorption in narrow finite slits is partially depressed in compared to the infinite ones. On the other hand, in the work of Yang et al., methane is represented in a simpler manner (LJ pair-wise interactions) than it is done with a NLDFT-SAFT formalism. The principal consequence is that the densities computed by Yang et al. are underestimated at high pressure and so is the solvation pressure. For instance, the maximum average density obtained in this work at ( $T=303 \mathrm{~K}, P=200$ bar) 


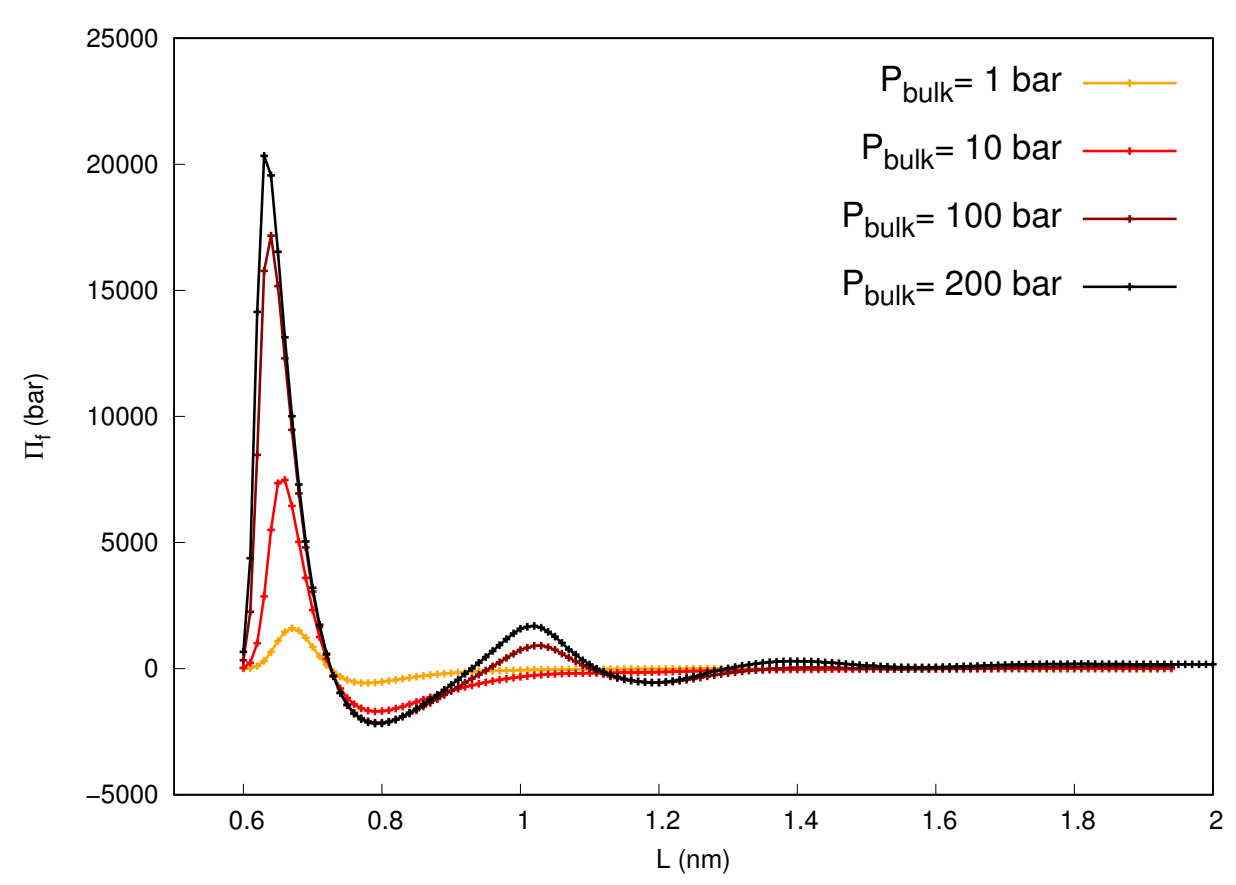

Fig. 5 Pore pressure evolution as a function of pore size for four different values of bulk pressure $\left(P_{\text {bulk }} \in\{1,10,100,200\}\right.$ bar $)$ at $373 \mathrm{~K}$.

is six times greater and consequently the solvation pressure ten times greater than the ones obtained by Yang et al. in similar conditions.

This oscillatory decay has been previously observed for the solvation pressure in liquids, theoretically and experimentally by different authors [20,48, 49,50]. Israelachvili 20] proposed an exponential oscillatory decay with a pseudo-period close to $\sigma_{f f}$ and a characteristic decay length equal to $\alpha \times \sigma_{f f}$ with $\alpha \in\{1.2 ; 1.7\}$ for liquids. Fig. 6 presents the evolution of the estimated solvation pressure with the normalised pore width and one can observe that the same type of exponential decay is obtained here at $T=373 \mathrm{~K}$ and $P_{\text {bulk }}=200 \mathrm{bar}$ for supercritical methane. A characteristic exponential decay factor of $\alpha=1.16$ is recovered here. Therefore, one can consider that for pore widths larger that $5 \times \alpha \times \sigma_{f f} \approx 2 \mathrm{~nm}$, the pore pressure does not oscillate anymore and that the bulk pressure is fully recovered. Moreover, Fig. 7 shows that the dependence of the characteristic exponential decay factor $\alpha$ with the temperature is quite linear. To the authors' knowledge, this was never reported in the literature but only three temperatures are investigated here and further results are needed to confirm this trend.

In addition, it is noteworthy that the extrema of the pore pressure do not exactly correspond to the extrema of the average density in the pore (see Fig. 8). Indeed, such a correspondence should only happen in the case of hard walls as observed by Henderson [48] and the slight difference of position between the extrema of pore pressure and average density is a result of the fluid-solid potential $V^{\text {ext }}$. In the literature, the maxima of $\Pi_{f}$ are often attributed to the maxima of density occurring for the most ordered states in commensurate pores and the minima for 


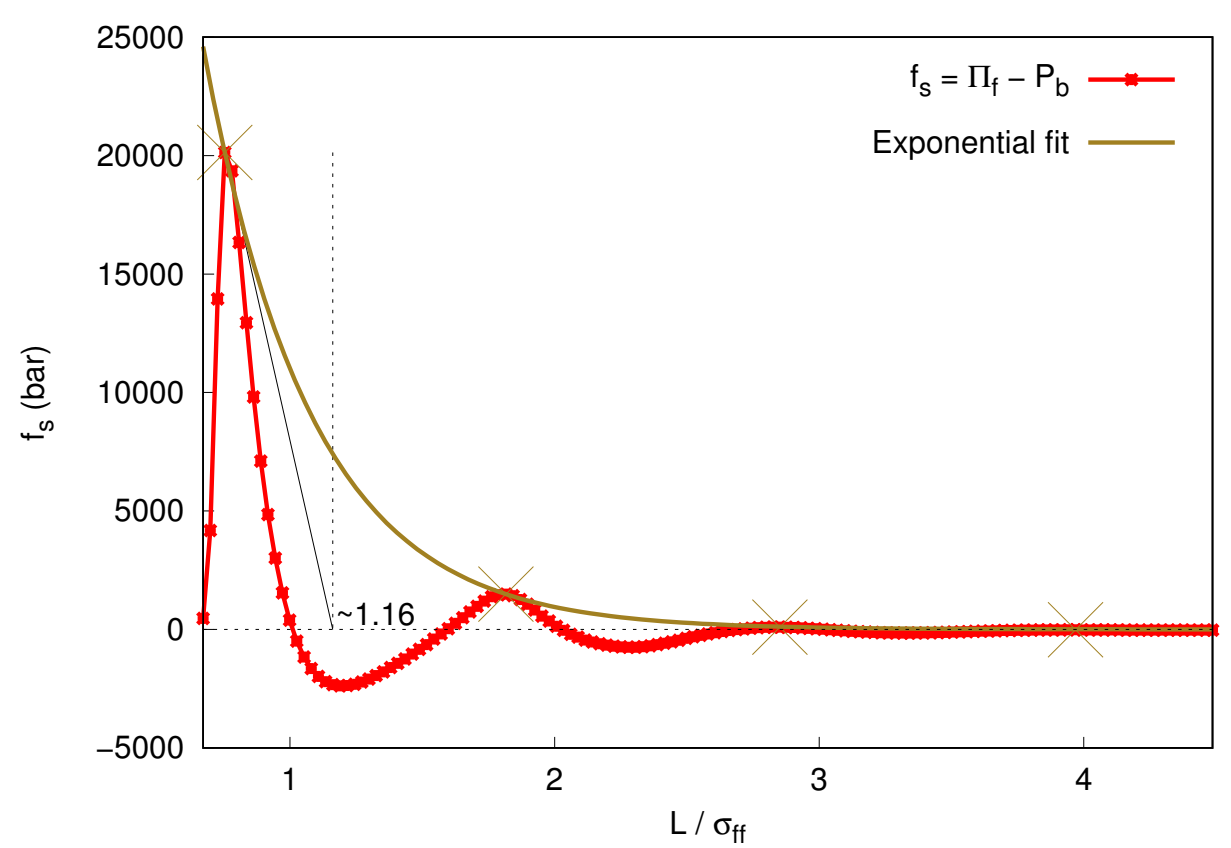

Fig. 6 Solvation pressure evolution as a function of normalised pore widths at $T=373 \mathrm{~K}$ and $P_{\text {bulk }}=200$ bar.

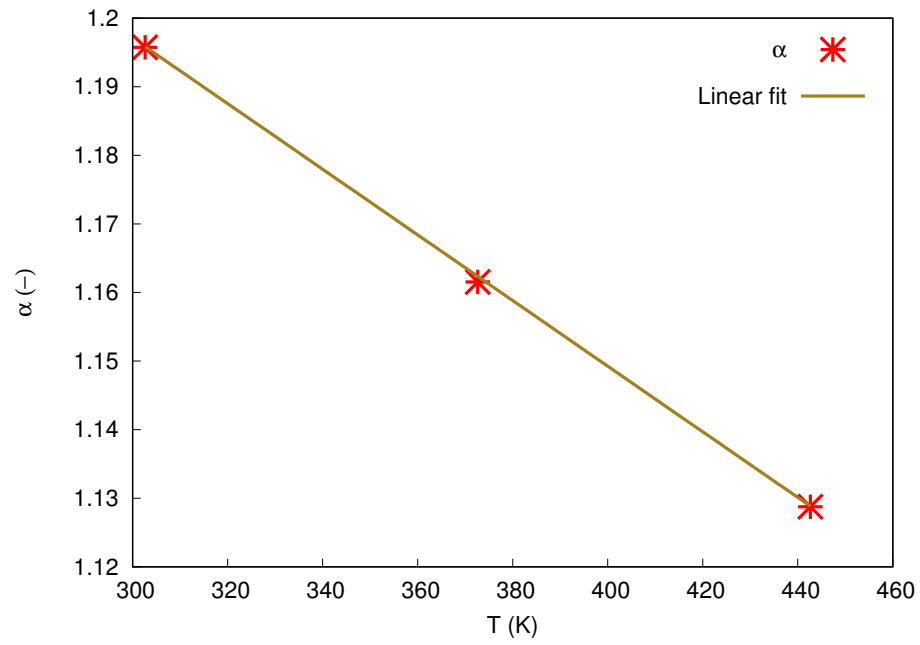

Fig. 7 Evolution of the characteristic exponential decay factor with the temperature at $P_{\text {bulk }}=200$ bar. 
the least ordered states in non-commensurate pores [20,22] maybe as a result of less complete maps of pore pressure vs pore widths. This emphasizes the usefulness of the calculation speed of DFTs. Fig. 8 highlights also the influence of temperature for the three different temperatures considered here $(T \in\{443,373,303\} \mathrm{K})$. It has already been shown in the literature [51 that the density profiles are less structured and the mean density maxima are less pronounced when temperature increases. This behaviour may be attributed to the increase of kinetics contribution in the free energy. We can observe here exactly the same behaviour on the pore pressure maxima. With increasing temperature, they are less and less pronounced. Moreover one can notice that the shift between pore pressure and mean density profiles also decreases with increasing temperature. This means that this shift is mostly driven by short range interactions whose influence decreases when temperature and thus molecular agitation increase.

In order to have a better understanding of the relation between the pore density profiles and the induced attractive or repulsive pore pressure, it is useful to compute the work of the pore pressure as this latter allows to distinguish stable and unstable configurations, as shown in Fig. 9. Hence, it is demonstrated here that the pore pressure work can be used to identify unambiguously the pore widths that are commensurate (or non-commensurate) with the size of an adsorbent.

\section{Concluding remarks}

In this paper, we have shown that:

- A non-local density functional theory can be used to obtain highly resolved 3D maps of pore pressure versus pore widths and bulk pressure of methane in graphitic slit-shaped pores at different temperatures.

- These maps clearly highlight that, even for a non polar fluid such as methane, the pore pressure reaches very high values in the narrowest nanopores and is a complex function of both pore width and thermodynamic conditions.

- For ultramicropores accommodating only one fluid layer, the pores exhibit only positive pore pressure, and so positive adsorption stress causing expansion. For larger pores accommodating at least two overlapping layers, one can observe a transition from initial negative pore pressures to positive ones with increasing bulk pressures. This behaviour reflects the competition between fluid-solid interactions - predominant at low pressure - and fluid-fluid interactions - that dominate at higher pressure.

- For a given bulk pressure and with increasing pore widths, an oscillatory decay of the pore pressure is recovered giving successively repulsive or attractive force on the surface of the solids walls. The dependence of the characteristic exponential decay factor is linear with temperature in the range analysed here and is the order of $1.2 \times \sigma_{f f}$.

- It has been also clearly highlighted that the pore pressure maxima differ from the mean density ones.

- This oscillatory decay reflects both the molecular packing and the fluid confinement. The pore pressure work distinguishes stable and unstable configurations. Therefore, it can be used to unambiguously identify the pore widths that are commensurate (or not-commensurate) with the size of an adsorbent. 

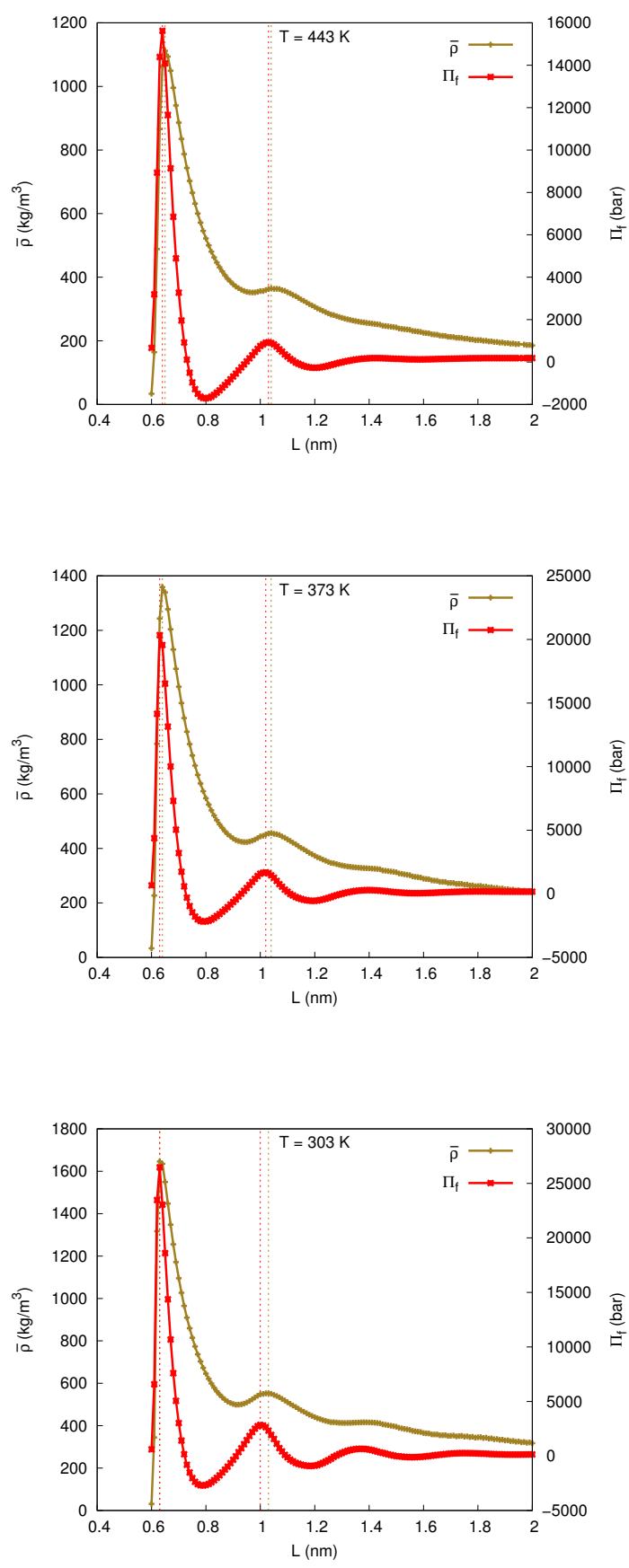

$$
\bar{\rho}=\frac{1}{L^{*}} \int_{0}^{L} \rho(z) d z \quad \text { with } \quad L^{*}=L-2 e \quad \text { and } \quad e=0.79 \times \sigma_{s f}
$$

Fig. 8 Comparison between the pore pressure evolution and the average pore density evolution as a function of pore size for three different temperatures $(T \in\{443,373,303\} \mathrm{K})$ at 200 bar. 

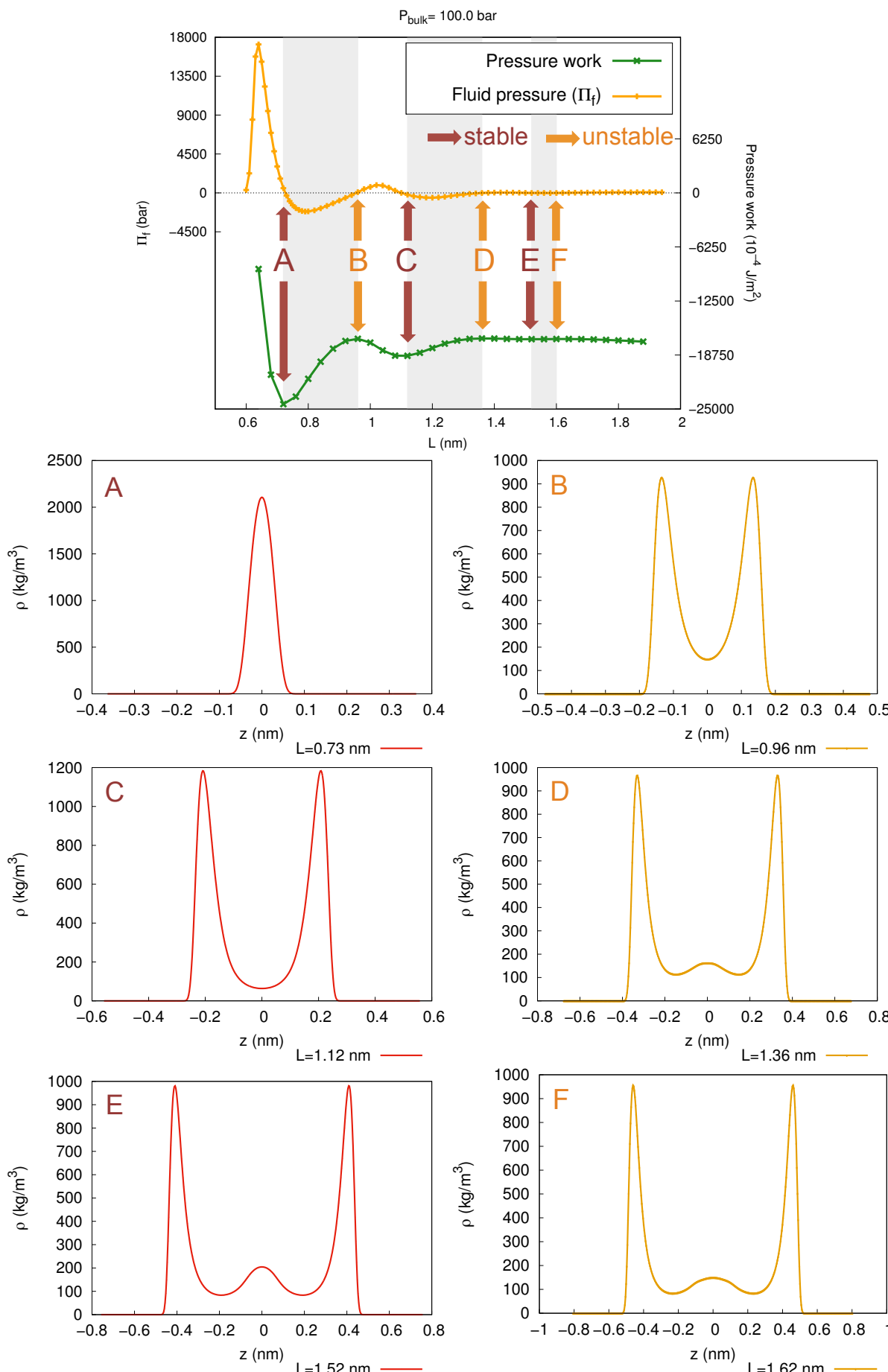

Fig. 9 Pore pressure work evolution as a function of pore widths (top) and characteristic pore density profiles (bottom, A to F) at $373 \mathrm{~K}$. 
These conclusions emphasise the need to have a reliable continuous thermodynamic model with a low computational cost to compute the pore fluid properties and their impact on the solid matrix in order to be coupled with a poromechanical model. A non-local DFT coupled with the SAFT equation-of-state can play this role for complex fluids on wide thermodynamic conditions.

\section{Supplementary Materials}

Animated versions of Fig. 10 .

Acknowledgements Financial supports from the Communauté de Communes de Lacq-Orthez and Conseil Départemental 64 are gratefully acknowledged. We also gratefully acknowledge Dr. Mourad Abouzaid for his advices and our different discussions, especially concerning the mathematical developments.

\section{References}

1. K.S.W. Sing, D.H. Everett, R.A.W. Haul, L. Moscou, R.A. Pierotti, J. Rouquerol, T. Siemieniewska, Pure and Applied Chemistry 57(4), 603 (1985)

2. M. Thommes, K. Kaneko, A.V. Neimark, J.P. Olivier, F. Rodriguez-Reinoso, J. Rouquerol, K.S.W. Sing, Pure and Applied Chemistry 87(9-10), 1051 (2015)

3. J.W. Larsen, International Journal of Coal Geology $\mathbf{5 7}(1), 63$ (2004)

4. Z. Pan, L. Connell, International Journal of Coal Geology 69(4), 243 (2007)

5. J.R. Levine, Geological Society, London, Special Publications 109(1), 197 (1996)

6. M. Thommes, K.a. Cychosz, Adsorption 20, 233 (2014)

7. G.Y. Gor, P. Huber, N. Bernstein, Applied Physics Reviews 4(1), 11303 (2017)

8. F.T. Meehan, Proceedings of the Royal Society A: Mathematical, Physical and Engineering Sciences 115(770), 199 (1927)

9. H. Briggs, R.P. Sinha, Proceedings of the Royal Society of Edinburgh 53, 48 (1933)

10. S. Day, R. Fry, R. Sakurovs, International Journal of Coal Geology 74(1), 41 (2008)

11. S. Ottiger, R. Pini, G. Storti, M. Mazzotti, Adsorption 14(4-5), 539 (2008)

12. R. Pini, S. Ottiger, L. Burlini, G. Storti, M. Mazzotti, Journal of Geophysical Research: Solid Earth (1978-2012) 114(B4) (2009)

13. S. Hol, C.J. Spiers, Journal of the Mechanics and Physics of Solids 60(11), 1862 (2012)

14. D.N. Espinoza, M. Vandamme, J.M. Pereira, P. Dangla, S. Vidal-Gilbert, International Journal of Coal Geology 134, 80 (2014)

15. L. Perrier, F. Plantier, D. Grégoire, Review of Scientific Instruments 88 (2017)

16. L. Perrier, Coupling between adsorption and deformation in microporous media. Ph.D. thesis, Universit \{́́\} Pau \& Pays Adour (2015)

17. M.A. Biot, Journal of Applied Physics 12(2), 155 (1941)

18. O. Coussy, Poromechanics (John Wiley $\{\&\}$ Sons, Ltd., 2004)

19. L. Perrier, G. Pijaudier-Cabot, D. Grégoire, Continuum Mechanics and Thermodynamics 27(1-2), 195 (2015)

20. J.N. Israelachvili, Intermolecular and surface forces (Academic press, 2015)

21. D.D. Do, D. Nicholson, H.D. Do, Journal of Physical Chemistry C 112(36), 14075 (2008)

22. R. Diao, C. Fan, D.D. Do, D. Nicholson, The Journal of Physical Chemistry C 120(51), 29272 (2016)

23. V.T. Nguyen, D.D. Do, D. Nicholson, Journal of Colloid and Interface Science 388(1), 209 (2012)

24. P. Kowalczyk, S. Furmaniak, P.A. Gauden, A.P. Terzyk, Journal of Physical Chemistry C 114(11), $5126(2010)$

25. P. Kowalczyk, C. Balzer, G. Reichenauer, A.P. Terzyk, P.A. Gauden, A.V. Neimark, Carbon 103, $263(2016)$

26. P. Kowalczyk, A. Ciach, A.P. Terzyk, P.A. Gauden, S. Furmaniak, Journal of Physical Chemistry C 119(11), 6111 (2015) 
27. F. Khaddour, A. Knorst-Fouran, F. Plantier, M.M. Piñeiro, B. Mendiboure, C. Miqueu, Adsorption 20(4), 649 (2014)

28. R. Vermorel, G. Pijaudier-Cabot, European Journal of Mechanics-A/Solids 44, 148 (2014)

29. L. Perrier, G. Pijaudier-Cabot, D. Grégoire, Journal of the Mechanics and Physics of Solids - (to be submitted, 2017)

30. R. Evans, in Fundamentals of Inhomogeneous Fluids, ed. by D. Henderson (Marcel Dekker Inc., New York, NY, 1992), chap. Density Fu, pp. 85-176

31. H.T. Davis, Statistical mechanics of phases, interfaces and thin films. (Wiley-VCH, NewYork, 1998)

32. C. Malheiro, B. Mendiboure, F. Plantier, F.J. Blas, C. Miqueu, Journal of Chemical Physics 140, 134707 (2014)

33. C. Malheiro, B. Mendiboure, J.M. Miguez, M.M. Piñeiro, C. Miqueu, The journal of physical chemistry. C 118(43), 24905 (2014)

34. P.B. Balbuena, D. Berry, K.E. Gubbins, The Journal of Physical Chemistry 97(4), 937 (1993)

35. P.I. Ravikovitch, A.V. Neimark, Langmuir 22(26), 10864 (2006)

36. C. Balzer, R.T. Cimino, G.Y. Gor, A.V. Neimark, G. Reichenauer, Langmuir 32(32), 8265 (2016)

37. K. Yang, Y. Lin, X. Lu, A.V. Neimark, Journal of Colloid and Interface Science 362(2), $382(2011)$

38. E. Ustinov, D. Do, Carbon 44(13), 2652 (2006)

39. K. Yang, X. Lu, Y. Lin, A.V. Neimark, Energy and Fuels 24(11), 5955 (2010)

40. A. Gil-Villegas, A. Galindo, P.J. Whitehead, S.J. Mills, G. Jackson, A.N. Burgess, Journal of Chemical Physics 106(10), 4168 (1996)

41. H. Hansen-Goos, R. Roth, Journal of physics. Condensed matter : an Institute of Physics journal 18(37), 8413 (2006)

42. Y.X. Yu, J. Wu, The Journal of Chemical Physics 116(16), 7094 (2002)

43. F. Llovell, A. Galindo, F.J. Blas, G. Jackson, Journal of Chemical Physics 133(2) (2010)

44. W.A. Steele, Surface Science 36(1), 317 (1973)

45. Z. Tan, K.E. Gubbins, Journal of Physical Chemistry 94(15), 6061 (1990)

46. J.P. Hansen, I.R. McDonald, Theory of Simple Liquids (Third Edition) (Elsevier, 2006)

47. I.V. Kopanichuk, A.A. Vanin, E.N. Brodskaya, Colloids and Surfaces A: Physicochemical and Engineering Aspects 527(May), 42 (2017)

48. J.R. Henderson, Molecular Physics 59(1), 89 (1986)

49. P. Tarazona, L. Vicente, Molecular Physics 56(3), 557 (1985)

50. H.K. Christenson, J. Chem. Phys. 78(11), 6906 (1983)

51. T.X. Nguyen, S.K. Bhatia, D. Nicholson, Journal of Chemical Physics 117(23), 10827 (2002)

\section{A Development of the pore pressure estimation}

In the context of a slit pore of width $L$ and surface $A_{0}$, filled with pure methane, Eqs. 11 and 13 may be rewritten as:

$$
\begin{aligned}
& \qquad(\rho)=A(\rho)-A_{0} \int_{-L / 2}^{L / 2} \rho(z)\left(\mu_{b}-V^{e x t}(z)\right) d z \\
& \text { and } \quad \Pi_{f}=-\frac{1}{A_{0}}\left(\frac{\partial \Omega}{\partial L}\right)_{T, A_{0}, \mu} .
\end{aligned}
$$

Therefore,

$$
\Pi_{f}=-\frac{1}{A_{0}}\left(\frac{\partial A}{\partial L}\right)_{T, \mu, A_{0}}+\underbrace{\frac{\partial}{\partial L} \int_{-L / 2}^{L / 2} \rho(z, L)\left(\mu_{b}-V^{e x t}(z, L)\right) d z}_{I_{1}}
$$


On one hand, considering $\mu$ as a constant during the process of the partial derivation by $L$, we get:

$$
\begin{aligned}
\left(\frac{\partial A}{\partial L}\right)_{T, \mu, A_{0}} & =0 \\
\text { and }\left(\frac{\partial \rho}{\partial L}\right)_{T, \mu, A_{0}} & =0
\end{aligned}
$$

On a second hand, considering the substitution $z=\varphi(u)=u L$, we get:

$$
\begin{aligned}
& \Pi_{f}=I_{1} \underset{u=\frac{z}{L}}{=} \frac{\partial}{\partial L} \int_{-1 / 2}^{1 / 2} \rho(u L, L)\left[\mu_{b}-V^{e x t}(u L, L)\right] L d u \\
& =\int_{-1 / 2}^{1 / 2} \frac{\partial}{\partial L}\left\{\rho(u L, L)\left[\mu_{b}-V^{e x t}(u L, L)\right] L\right\} d u \\
& =\underbrace{\int_{-1 / 2}^{1 / 2} \frac{\partial}{\partial L}\left[\rho(u L, L) \mu_{b} L\right] d u}_{I_{2}}-\underbrace{\int_{-1 / 2}^{1 / 2} \frac{\partial}{\partial L}\left[\rho(u L, L) V^{e x t}(u L, L) L\right] d u}_{I_{3}} . \\
& I_{2}=\underbrace{\int_{-1 / 2}^{1 / 2} \rho(u L, L) \mu_{b} d u}_{I_{4}}+\underbrace{\int_{-1 / 2}^{1 / 2} \mu_{b} L \frac{\partial \rho(u L, L)}{\partial L} d u}_{I_{5}}
\end{aligned}
$$

where

$$
I_{4} \underset{z=u L}{=} \int_{-L / 2}^{L / 2} \frac{\rho(z, L) \mu_{b}}{L} d z=\frac{\mu_{b}}{L} \int_{-L / 2}^{L / 2} \rho(z, L) d z
$$

and

$$
\begin{aligned}
I_{5} & =\int_{-1 / 2}^{1 / 2} \mu_{b} L \frac{\partial \rho(u L, L)}{\partial L} d u=\int_{-1 / 2}^{1 / 2} \mu_{b} L\left(u \frac{\partial \rho(u L, L)}{\partial z}+\frac{\partial \rho(u L, L)}{\partial L}\right) d u \\
z=u L & \int_{-L / 2}^{L / 2} \mu_{b} \not L\left(\frac{z}{L} \frac{\partial \rho(z, L)}{\partial z}+\frac{\partial \rho(z, L)}{\partial L}\right) \frac{d z}{\not \nvdash} \\
& =\mu_{b} \int_{-L / 2}^{L / 2} \frac{z}{L} \frac{\partial \rho(z, L)}{\partial z} d z+\mu_{b} \int_{-L / 2}^{L / 2} \underbrace{\frac{\partial \rho(z, L)}{\partial L}}_{\text {see Eq. 19 })} d z \\
\text { by parts } & \mu_{b}\left[\frac{z \rho}{L}\right]_{-L / 2}^{L / 2}-\mu_{b} \int_{-L / 2}^{L / 2} \frac{\rho(z, L)}{L} d z \\
= & -\frac{\mu_{b}}{L} \int_{-L / 2}^{L / 2} \rho(z, L) d z .
\end{aligned}
$$


Therefore

$$
\begin{gathered}
I_{2}=I_{4}+I_{5}=0 . \\
\text { - } I_{3}=\underbrace{\int_{-1 / 2}^{1 / 2} \rho(u L, L) V^{e x t}(u L, L) d u}_{I_{7}}+\underbrace{\int_{-1 / 2}^{1 / 2} V^{e x t}(u L, L) L \frac{\partial \rho(u L, L)}{\partial L} d u}_{I_{8}} \\
+\underbrace{\int_{-1 / 2}^{1 / 2} \rho(u L, L) L \frac{\partial V^{e x t}(u L, L)}{\partial L} d u}_{I_{9}}
\end{gathered}
$$

where

$$
\begin{aligned}
& I_{7} \underset{z=u L}{=} \int_{-L / 2}^{L / 2} \frac{\rho(z, L) V^{e x t}(z, L)}{L} d z \\
& I_{8}=\int_{-1 / 2}^{1 / 2} V^{e x t}(u L, L) L\left(u \frac{\partial \rho(u L, L)}{\partial z}+\frac{\partial \rho(u L, L)}{\partial L}\right) d u \\
& \underset{z=u L}{=} \int_{-L / 2}^{L / 2} V^{e x t}(z, L) L(\frac{z}{L} \frac{\partial \rho(z, L)}{\partial z}+\underbrace{\frac{\partial \rho(z, L)}{\partial L}}_{\text {see Eq. 19. }}) \frac{d z}{L} \\
& \text { by parts }\left[\frac{z V^{e x t} \rho}{L}\right]_{-L / 2}^{L / 2}-\int_{-L / 2}^{L / 2} \frac{\rho V^{e x t}}{L} d z-\int_{-L / 2}^{L / 2} \frac{z}{L} \rho \frac{\partial V^{e x t}}{\partial z} d z
\end{aligned}
$$

and

$$
I_{9} \underset{z=u L}{=} \int_{-L / 2}^{L / 2} \rho(z, L) z \frac{\partial V^{e x t}(z, L)}{\partial z} \frac{d z}{L}+\int_{-L / 2}^{L / 2} \rho(z, L) \frac{\partial V^{e x t}(z, L)}{\partial L} d z .
$$

Therefore

$$
I_{3}=I_{7}+I_{8}+I_{9}=\int_{-L / 2}^{L / 2} \rho(z, L) \frac{\partial V^{e x t}(z, L)}{\partial L} d z
$$

and

$$
\Pi_{f}=I_{1}=I_{2}-I_{3}=-\int_{-L / 2}^{L / 2} \rho(z, L) \frac{\partial V^{e x t}(z, L)}{\partial L} d z
$$


For a symmetrical external potential such as the Steele-type potential (see Eq. 111), we get also:

$$
\begin{aligned}
\Pi_{f} & =-\int_{0}^{L} \rho(z, L) \frac{\partial V^{\text {ext }}(z, L)}{\partial L} d z \\
& =-\int_{0}^{L} \rho(z, L) \frac{\left.\partial\left(V^{\text {steele }}(z)+V^{\text {steele }}(L-z)\right)\right)}{\partial L} d z \\
& =-\int_{0}^{L} \rho(z, L) \frac{\partial\left(V^{\text {steele }}(L-z)\right)}{\partial L} d z \\
& =-\int_{0}^{L} \rho(z, L) \frac{\partial V^{\text {steele }}}{\partial z}(L-z) d z \\
& =-\int_{0}^{L} \rho(z, L) \frac{\partial V^{\text {steele }}}{\partial z}(z) d z .
\end{aligned}
$$

Finally

$$
\Pi_{f}=-\int_{0}^{L} \rho(z, L) \frac{\partial V^{\text {steele }}}{\partial z}(z) d z
$$

B Pore pressure 3D-maps at $303 \mathrm{~K}, 373, \mathrm{~K}$ and $443 \mathrm{~K}$ 

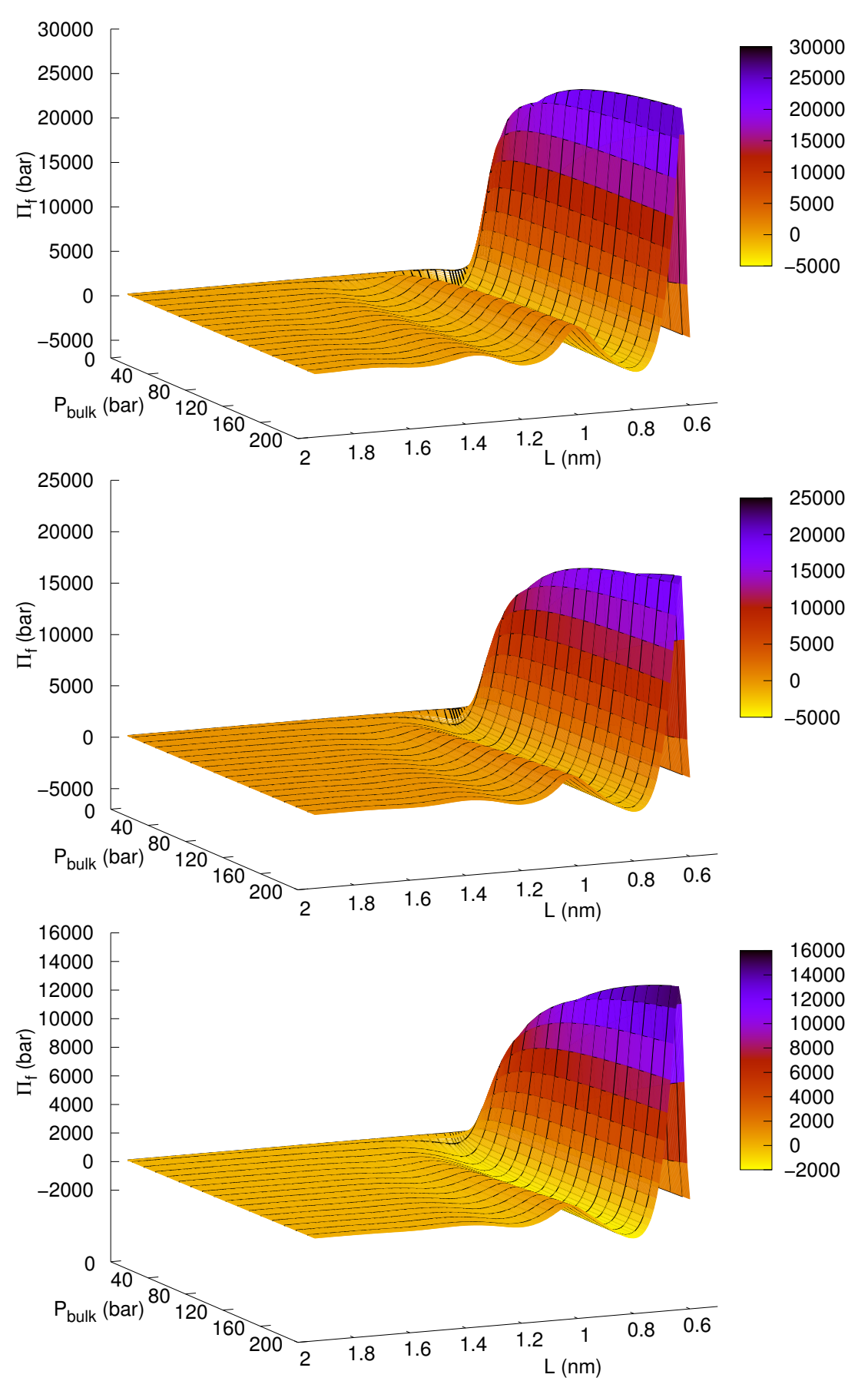

Fig. 10 Pore pressure as a function of bulk pressure and pore size at $303 \mathrm{~K}$ (top), $373 \mathrm{~K}$ (center) and $443 \mathrm{~K}$ (bottom). 